Published in final edited form as:

Eur J Cancer. 2020 February ; 126: 78-90. doi:10.1016/j.ejca.2019.12.006.

\title{
PET-based staging reduces the prognostic impact of early disease progression in patients with follicular lymphoma
}

Connie L. Batlevi, MD, PhD ${ }^{1,{ }^{*}}$, Fushen Sha, MD ${ }^{1,{ }^{*}}$, Anna Alperovich, $\mathrm{MD}^{1,{ }^{*}}$, Ai Ni, $\mathrm{PhD}^{2}$, Katy Smith, MBChB BSc ${ }^{1}$, Zhitao Ying, MD ${ }^{1}$, John F. Gerecitano, MD, PhD ${ }^{1}$, Paul A. Hamlin, MD¹, Steve M. Horwitz, MD ${ }^{1}$, Erel Joffe, MD ${ }^{1}$, Anita Kumar, MD ${ }^{1}$, Matthew J. Matasar, MD ${ }^{1}$, Alison J. Moskowitz, MD ${ }^{1}$, Craig H. Moskowitz, MD ${ }^{1}$, Ariela Noy, MD ${ }^{1}$, Colette Owens, MD ${ }^{1}$, M. Lia Palomba, MD ${ }^{1}$, David Straus, MD ${ }^{1}$, Gottfried von Keudell, MD ${ }^{1}$, Andrew D. Zelenetz, MD, $\mathbf{P h D}^{1}$, Venkatraman E Seshan, PhD $^{2}$, Stefano Luminari, MD ${ }^{3,4}$, Luigi Marcheselli, MS ${ }^{5}$, Massimo Federico, MD ${ }^{3}$, Anas Younes, MD ${ }^{1}$

${ }^{1}$ Department of Medicine, Lymphoma Service, Memorial Sloan Kettering Cancer Center, New York, NY

${ }^{2}$ Department of Epidemiology and Biostatistics, Memorial Sloan Kettering Cancer Center, New York, NY

${ }^{3}$ Department of Surgical, Medical, Dental and Morphological Sciences related to Transplant, Oncology and Regenerative medicine, University of Modena and Reggio Emilia, Italy.

${ }^{4}$ Hematology Unit, AUSL IRCCS Reggio Emilia, Reggio Emilia, Italy

${ }^{5}$ Department of Medical and Surgical Sciences, and FIL Trial Office, University of Modena and Reggio Emilia, Italy.

\section{Summary}

Background-Previous studies reported that early progression of disease (POD) after initial therapy predicted poor overall survival (OS) in patients with follicular lymphoma (FL). Here, we investigated whether pre-treatment imaging modality had an impact on prognostic significance of POD.

Corresponding Author: Connie Batlevi, MD, PhD, Memorial Sloan Kettering Cancer Center, Box 330, 1275 York Ave, New York,

NY, 10065, Phone: 212-639-8081, leec@mskcc.org.

*Authors contributed equally to this work

Katy Smith: The Royal Marsden Hospital, London, United Kingdom

Zhitao Ying: Peking University Cancer Hospital, Beijing, China

Craig H. Moskowitz: Sylvester Comprehensive Cancer Center, University of Miami Health System, Miami, FL.

Andy Ni: Division of Biostatistics, College of Public Health, Ohio State University, OH.

Contributors

Conception and design: C.L.B., F.S., A.A., A.Y.; Collection and assembly of data: C.L.B., A.A., F.S., K.S., Z.Y., S.L., L.M., M.F.;

Data analysis and interpretation: C.L.B., A.Y., F.S., V.E.S., A.N., A.A., S.L., L.M., M.F.; Manuscript writing: C.L.B., F.S., A.A., A.Y. contributed to writing the first draft of the manuscript. All authors provided a critical review of the manuscript's content and approved the final manuscript.

Publisher's Disclaimer: This is a PDF file of an unedited manuscript that has been accepted for publication. As a service to our customers we are providing this early version of the manuscript. The manuscript will undergo copyediting, typesetting, and review of the resulting proof before it is published in its final form. Please note that during the production process errors may be discovered which could affect the content, and all legal disclaimers that apply to the journal pertain. 
Methods-In this retrospective study, we identified 1,088 patients with grade 1-3A FL of whom 238 patients with stage II-IV disease were initially treated with R-CHOP and 346 patients treated with R-chemotherapy. Patients ( $\mathrm{N}=484)$ from the FOLL05 study served as an independent validation cohort. We risk-stratified patients based on pre-treatment radiographic imaging (PET vs. $\mathrm{CT}$ ) and early POD status using event defining and landmark analyses. A competing risk analysis evaluated the association between early POD and histologic transformation.

Findings-In the discovery cohort, patients with POD within 24 months (PFS24) of initiating RCHOP therapy had 5-year OS of 57.6\% for CT-staged patients compared with 70.6\% for PETstaged patients. In the validation cohort, the 5-year OS for patients with early POD was 53.9\% and $100 \%$ in CT- and PET-staged patients, respectively. The risk of histologic transformation in patients whose disease progressed within one year of initiating therapy was higher in CT-staged patients compared with PET-staged patients (16.7\% vs. 6.3\%), which was associated with a 9.7 fold higher risk for death.

Interpretation-In FL, pre-treatment PET-staging reduced the prognostic impact of early POD compared with CT-staging. Patients with early POD and no histologic transformation have an extended OS with standard therapy.

Funding-NCI Cancer Center Support Grant (P30 CA008748)

\section{Keywords}

PET; follicular lymphoma; early progression; PFS24; R-CHOP

\section{Introduction}

Follicular lymphoma (FL) is the second most common lymphoma in the United States, compromising approximately $30 \%$ of all lymphomas.[1,2] As treatment outcomes and overall survival (OS) continue to improve, surrogate endpoints are increasingly needed to predict OS in prospective clinical trials.

Previous studies proposed early progression or an early event were surrogate endpoints predicting poor OS in patients with newly diagnosed FL. In one study, FL patients whose disease progressed within 24 months from diagnosis (progression-free survival at 24 months; PFS24) after treatment with rituximab, cyclophosphamide, doxorubicin, vincristine, and prednisone (R-CHOP) had a 5-year OS of 50\%, compared with $90 \%$ for those without early progression of disease (POD).[3] In a different study, patients with an event within 12 months (event-free survival at 12 months; EFS12) after treatment with immunochemotherapy also had a poor OS.[4] These two studies shared similar but distinct definitions of early POD. One study based the analysis on event free survival, which included start of new lymphoma therapy as an event, while the other utilized progression free survival therefore early POD lacks a common definition. Nonetheless, the studies identified patients with early POD as a subgroup of FL patients with inferior outcomes and represented an area of unmet medical needs. These observations suggested utilization of a new clinical endpoint to evaluate new treatment strategies possibly leading to rapid approval by regulatory agencies. Furthermore, these observations suggested more intensive treatment 
approaches, including salvage chemoimmunotherapy followed by consolidative autologous stem cell transplantation, may be needed to improve treatment outcome. [5-7]

However, the imaging modality used at diagnosis and prior to treatment initiation in the earlier studies was not described.[3, 4] Whether the prognostic significance is maintained in the PET-based staging era is currently unknown. Positron emission tomography with 2-[ $\left.{ }^{18} \mathrm{~F}\right]$-Flouro-2-deoxyglucose integrated with computed tomography (FDG-PET, hereafter PET) has emerged as an important imaging tool for staging, response assessment, and predicting treatment outcomes of FL.[8] PET imaging is more sensitive than computed tomography (CT) imaging in identifying extra-nodal disease and facilitates more accurate clinical staging or response assessment.[9-12] Furthermore, several studies highlight the ability of PET imaging to identify sites of suspected transformation for targeted biopsy, which may influence the choice of initial therapy and treatment outcome.[13-16]

With this background, we examined the impact of PET staging on treatment outcome and prognosis of FL. We also compared the prognostic significance of PFS24 after first-line therapy in FL patients whose disease was assessed by CT or PET imaging modalities.

\section{Methods}

\section{Study design}

This is a retrospective study of adult patients ( $\geq 18$ years old) diagnosed between the years of 1998 to 2009 with FL managed at Memorial Sloan Kettering Cancer Center (MSKCC). This timeframe was selected to include patients who were treated in the rituximab era and to allow adequate follow up. The institutional review board approved this study.

\section{Participants}

Patients with FL grade 3B, composite histology or de novo disease transformation at diagnosis, fewer than 3 clinic visits (indicative of a consultative role without long term follow up), or harboring an active concurrent malignancy were excluded. For the MSKCC cohort, pathology slides were confirmed at MSKCC. Radiographic modality used for staging was captured at both diagnosis and at time of first treatment. Transformation to DLBCL at time of relapse was confirmed with biopsy. From a database of 1,088 patients with follicular lymphoma (grade 1, 2 and $3 \mathrm{~A}$ ), we excluded 31 patients with histologic transformation to DLBCL prior to treatment, 164 patients were observed and did not require treatment. Eight hundred and ninety-three patients were evaluable at first treatment, of which 754 patients had stage II-IV disease (Figure 1). We identified 346 patients with stage II-IV grade 1-3A FL treated with rituximab and chemotherapy for analysis of the impact of PFS24 status on OS, 118 patients with pretreatment CT, and 228 patients with pretreatment PET (Figure 1). A subgroup of 238 patients with stage II-IV grade 1-3A FL who were initially treated with R-CHOP chemotherapy, 84 patients with pretreatment CT, and 154 patients with pretreatment PET, were identified for separate analysis. An independent cohort of $484 \mathrm{FL}$ patients treated with rituximab-based chemotherapy regimens between 2006 and 2010 in the prospective FOLL05 clinical trial conducted by the Fondazione Italiana Linfomi was used for validation.[17, 18] From the FOLL05 study, 161 FL patients treated with R-CHOP, 114 
patients with pretreatment $\mathrm{CT}$, and 47 patients with pretreatment PET, were identified for replication.

\section{Statistical analysis}

PFS24 failures were defined as patients with disease progression within 24 months of treatment initiation (PFS24). PFS24 achievers were defined as patients without disease progression within 24 months of treatment initiation. We selected time of initiating therapy instead of time of diagnosis as our landmark because our population included patients who were initially managed with observation but subsequently required therapy. Two methods were used independently to risk-stratify patients based on early progression status. First, we conducted an event defining analysis to directly compare with prior methods used by Casulo et al and Maurer et al.[3, 4] Accordingly, OS for PFS24 failures was defined as the time from PFS24 failure to death or last follow-up. OS for the PFS24 achievers were defined as the survival time from achieving PFS24 (i.e. 24 months post treatment initiation) until death or last follow-up. Patients were excluded from this analysis if they died within 24 months without disease progression or were lost to follow up within 24 months of first-line therapy. Secondly, we used a landmark analysis proposed by Anderson JR, et.al 1983 to compare the outcomes of PFS24 failures and the PFS24 achievers.[19] Using a common time origin for both groups allows comparison of outcomes to be more clinically interpretable and eliminates immortal time bias which arises when an event such as relapse occurs during the time of follow up.[19] OS in the landmark analysis was calculated from 24 months post treatment initiation to last follow up or death. Patients were excluded from the landmark analysis if they died from any cause or were lost to follow up within 24 months of starting first-line therapy. Kaplan-Meier methods were used to estimate survival probability.

To understand the association between early progression and transformation we conducted a competing risks analysis for patients treated with R-CHEMO in which a patient can experience one of two different events - transformation or death without transformation. The time origin was set at the time of progression or two years for patients who were progression-free for two years. The cause-specific event rates were estimated for three groups of patients, those who progress in the first year, second year, or progression free in the first 2 years. The rate of death without transformation is compared to the survival rates from risk defining event to quantify the rates of death following transformation within the time interval of interest. [20]

Continuous variables were summarized by median and range. Categorical variables were summarized by frequency and percentage. OS and PFS were estimated by Kaplan-Meier method and compared by log-rank tests. All tests are two-sided. P values less than or equal to 0.05 were considered statistically significant. All statistical analyses were conducted in $\mathrm{R} 3 \cdot 5 \cdot 0 .{ }^{[21]}$

\section{Role of the funding source}

The funder of the study had no role in study design, data collection, data analysis, data interpretation, or writing of the report. The corresponding author had full access to all the data in the study and had final responsibility for the decision to submit for publication. 


\section{Results}

The median age of all 1088 patients was 57 years (range 20-94). The median follow-up of the population was 8.3 years (range $0.2-17.5$ ), 9.5 years (range $0.3-17.5$ ) for patients staged with CT, and 7.9 years (range $0 \cdot 2-17 \cdot 2$ ) for patients staged with PET. There was a trend of increased utilization of PET imaging over time (Supplemental Figure 1).

In the MSKCC cohort, patients were unevaluable if they had incomplete radiographic records $(\mathrm{N}=57)$, missing treatment data $(\mathrm{N}=1)$, deceased within 24 months of treatment without progression $(\mathrm{N}=4)$, lost to follow up $(\mathrm{N}=12)$, or started second-line treatment without progression ( $\mathrm{N}=11)$ (Figure 1). Of four patients who died within 24 months without progression, 1 patient died from treatment related infection while 3 patients died from causes unrelated to treatment or lymphoma. The 11 patients who started second-line therapy without progression included 9 patients who had suboptimal or stable responses to treatment with associated change of therapy and 2 patients who suffered treatment toxicity. A total of 346 patients in the MSKCC cohort who had stage II-IV disease and were treated with rituximab plus chemotherapy regimens were evaluable for PFS24 status (Figure 1, Table 1). Patient characteristics were similar to 754 patients with stage II-IV disease who required treatment in the MSKCC cohort (Supplemental Table 1). The median age of the 346 patients treated with R-chemotherapy was 56 years (range 24-80). Five percent of the patients had stage II disease while $95 \%$ had stage III or IV disease before treatment initiation. FLIPI risk score at the time of treatment initiation was available for $80 \%$ of the CT-imaged and $86 \%$ of the PET-imaged patients. A high Follicular Lymphoma International Prognostic Index (FLIPI) represented 45\% (154/346) of patients.

R-CHOP was the primary treatment regimen making up $71 \%$ ( $\mathrm{N}=84$ ) of CT-staged and $68 \%$ $(\mathrm{N}=154)$ of PET-staged patients. Patients not achieving PFS24 represented 30\% (N=35) of CT-imaged, and 28\% ( $\mathrm{N}=63)$ of PET-imaged patients.

The validation cohort consisted of 484 patients from the FOLL05 clinical trial treated with Rituximab in combination with chemotherapy (Table 1). The median follow-up for the validation cohort was 6.8 years (range $0 \cdot 1-9 \cdot 9$ ). Nine percent of patients had stage II disease and $91 \%$ of patients had stage III or IV disease at time of treatment. High FLIPI represented $37 \%$ of the population. Treatment was evenly distributed between R-CHOP (33\%, Rituximab, cyclophosphamide, doxorubicin, vincristine, and prednisone), R-CVP (33\%, Rituximab, cyclophosphamide, vincristine, and prednisone) and R-FM (34\%, Rituximab, fludarabine, and mitoxantrone). Patients not achieving PFS24 included $32 \%(\mathrm{~N}=110)$ in the CT-imaged group, and 25\% ( $\mathrm{N}=35)$ in the PET-imaged group.

We initially analyzed PFS24 based on pre-treatment radiographic staging in patients treated with R-CHOP using an event defining analysis to facilitate comparison to previously published studies.[3, 4] (Figure 2). In the MSKCC cohort, 238 evaluable patients with stage II-IV were treated with R-CHOP. PFS24 failure was observed in 24\% (20/84) in CT-imaged and 25\% (39/154) in PET-imaged patients. A replication cohort of 161 patients treated with R-CHOP in the FOLL05 study was also analyzed. PFS24 failure was associated with inferior OS in the MSKCC cohort regardless of modality of staging (Figure 2A). However, 
the impact of PFS24 failure on OS was lessened with PET-based radiologic staging (Figure 2A). In CT-imaged patients, PFS24 failures demonstrated an estimated 5- and 10-year OS of $57.6 \%$ (95\% CI 39-85) and 43.2\% (95\% CI 22-86), respectively. In contrast, patients achieving PFS24 demonstrated an estimated 5- and 10-year OS of 90.2\% (95\% CI 83-98), and $84.0 \%$ (95\% CI 75-94), respectively (Figure 2A). With PET-based imaging prior to initiating treatment, PFS24 failures demonstrated an estimated 5- and 10-year OS of 70.6\% (95\% CI 57-87) and 67.1\% (95\% CI 53-85), respectively (Figure 2A). Among patients achieving PFS24 imaged with PET, the estimated 5- and 10-year OS were 95.0 \% (95\% CI 91-99) and 83.6\% (95\% CI 73-96), respectively. The FOLL05 validation cohort confirmed the improvement of outcome in PET-imaged patients who failed PFS24 ( $P=0 \cdot 857$, Figure 2B).

We next applied a landmark analysis using a 24 months post treatment landmark, to evaluate the prognosis in patients who are alive but failed to achieve PFS24. This analysis excluded 7 patients from the MSK cohort and 3 patients from the FOLL05 cohort for progression prior to the 24 mo landmark. Applying this method, PET-based staging further blunted the impact of PFS24 status on OS, while failure to achieve PFS24 continued to predict a poor OS in the CT-staged patients (Figure 3A), In the CT-imaged patients treated with R-CHOP, PFS24 failures had an estimated 5- and 10-year OS of 63.2\% (95\% CI 44-92) and 47.4\% (95\% CI 24-93) respectively, while PFS24 achievers had an estimated 5- and 10-year OS of 90.2 (95\% CI 83-98) and 84.0\% (95\% CI 75-94), respectively (Figure 3A,). Patients with PETbased imaging and experienced PFS24 failures demonstrated an estimated 5-and 10-year OS of $80.8 \%$ (95\% CI 68-96), and 76.1\% (95\% CI 62-94), respectively (Figure 3A), compared with 95\% (95\% CI 91-99), and 83.6\% (95\% CI 73-96) in patients who achieved PFS24 (Figure 3A). Similar results were observed in the FOLL05 validation cohort (Figure 3B, Supplemental Table 3).

Next, we investigated whether the data obtained from R-CHOP could be generalized to other Rituximab-based chemotherapeutic regimens (R-Chemo). In the MSKCC cohort, we identified 330 patients treated with R-Chemo regimens who were evaluable for the landmark analysis, 16 patients were excluded for progression within the 24 mo landmark (Figure 4). In patients imaged with CT prior to treatment, the estimated 5-year OS in patients who exceeded PFS24 was 92.3\% (95\% CI, 86.5-98.4) compared with 62.0\% (95\% CI, 45.983.7) for those not achieving PFS24 (Figure 4A). In the PET-imaged patients, the estimated 5-year OS in patients who exceeded PFS24 was 94.2\% (95\% CI, 90.4-98.2) compared with 79.4\% (95\% CI, 68.7-91.7) for those who failed to achieve PFS24 (Figure 4A). The validation FOLL05 cohort included 473 evaluable patients treated with R-Chemo, 11 patients were excluded from the 24 mo landmark. In the CT-imaged patients, the estimated 5-year OS was 95.7\% (95\% CI, 92.9-98.5) for patients who exceeded PFS24 compared with 66.9\% (95\% CI, 57.6-77.7) for those who failed to achieve PFS24 (Figure 4B). In contrast, PET-imaged patients who exceeded PFS24 had an estimated 5-year OS of 95.6\% (95\% CI, 91.5-99.9) compared with $84.3 \%$ (95\% CI, 72.5-98.0) for those who failed to achieve PFS24 (Figure 4B, Supplemental Table 4). Patients treated with R-Chemo analyzed using the event defining analysis supported the pattern that PET-based staging blunted the poor prognostic effect of early progression in both the MSKCC and the FOLL05 validation cohorts (Supplemental Figure 2). 
Early disease transformation has been shown to negatively impact OS in patients with FL. [22-25] Therefore, we performed a competing risk analysis of transformation and early death to determine if early transformation after initial treatment is associated with poor outcomes (Figure 5). Patients were categorized into three groups based on their early progression status: 1) PFS24 achiever, 2) progression within 1-year post treatment, 3) progression between 1 and 2 years post treatment. In patients who achieve PFS24, the cumulative risk of biopsy proven transformation at 3 years was 3.4\% and all -cause mortality risk at 3 years was also $3.4 \%$, with $<1 \%$ attributed to transformation and $2.5 \%$ to nontransformation associated causes. For patients who progress within 1-year post treatment, the cumulative risk of biopsy proven transformation was $31.9 \%$ at 1 year and $42.4 \%$ at 3 years. The all-cause mortality risk was 33\% with almost $60 \%$ (18.6/33) of patient deaths attributed to transformation while $40 \%$ (14.4/33) of patient deaths were unrelated to transformation. Transformation is a significant contribution to progression and early death during the first two years post treatment (Table 2).

In patients staged with $\mathrm{CT}$ prior to treatment, the cumulative risk of transformation at the time of progression for patients who progressed within 1 year, and 1-2 years were $16.7 \%$ and $8.7 \%$, respectively. In contrast, patients staged with PET prior to treatment demonstrated a lower cumulative risk of transformation at the time of progression for patients who progressed within 1 year, and 1-2 years of 6.3\% and 3.2\%, respectively (Supplemental Table 5). This supports the hypothesis that PET imaging at diagnosis and treatment excludes patients with transformation which previously would have gone undetected by CT.

\section{Discussion}

Our study provides evidence of the importance of pre-treatment PET-based staging in the current management of FL. While early progression remains a predictor of poor outcomes in FL, the observed difference in outcome is blunted in the era of PET-based staging. Patients who fail to achieve PFS24 and are alive at 24 months without evidence of transformation, may enjoy a prolonged OS without the need for more intensive, and potentially more toxic, therapy. In the MSKCC cohort, less than $20 \%$ of patients received a stem cell transplant at any line of subsequent therapy. Thirty eight percent of stem cell transplant recipients harbored disease transformation. Consistent with our data, a recent study from M. D. Anderson Cancer Center of 342 patients with advanced-stage, grade 1-2 FL without histologic transformation during their disease course, demonstrated a 5-year OS $>75 \%$ regardless of PFS24 status.[26] The diverse treatment landscape using a variety of nonintensive, targeted therapy regimens similarly demonstrate favorable OS in patients with early disease progression but without histologic transformation.[27-34] For example, a recent update of the CHRONOS-1 study showed patients with relapsed or refractory FL without evidence of disease transformation treated with single-agent copanlisib demonstrated similar OS regardless of their PFS24 status.[35] A combined analysis of three clinical trials using rituximab-based doublets showed a significant but blunted impact of PFS24 on 5-year OS.[6] Therefore, caution should be used before considering PFS24 broadly without regards to transformation status as a surrogate endpoint for OS or applying this surrogate endpoint to evaluate the efficacy of new therapeutic agents. 
The discrepancy in OS between CT and PET-based imaging in patients with PFS24 failure is likely from a multitude of factors including improving prognosis afforded by recent advances as well as the ability of PET-based imaging to identify areas of disease transformation. With pre-treatment CT imaging alone, a diagnostic biopsy is typically obtained from a less invasive peripheral lymph node. This approach may miss other disease areas involved with de novo histologic transformation, which carries a poor prognosis. With pre-treatment PET imaging, disease sites with high FDG-PET uptake are typically biopsied, and if de novo disease transformation is found, those patients are typically excluded from FL-based therapy and databases. Accordingly, in our retrospective data analysis, de novo transformed FL were not included in the FL database, and this likely impacted the better OS in our report compared with other published series.

In our study, we demonstrate that patients who fail to achieve PFS24 after treatment and undergo histologic transformation suffered the highest risk of early mortality. In a retrospective pooled analysis with over 8,000 FL patients, the 5-year survival after transformation was $34 \%$ for patients experiencing early ( $\triangleleft$ year) histological transformation and $48 \%$ for those with late histological transformation.[25] In addition, a study by the British Columbia Cancer Agency showed that $77 \%$ of patients treated with rituximab and bendamustine with an early progression had a transformative event.[36] In the PRIMA trial, more than half (58\%) of the transformations were documented during the first year after induction, and $38 \%$ of the biopsies performed during the first year identified transformations.[22] These results support the hypothesis that the majority of transformations occur in patients with early progression after frontline treatment. Cooccurrence of early progression and transformation are major contributors to early mortality. Both studies incorporated subsets of patients with PET-imaging. Upon early recurrence, PET based imaging and biopsy is warranted to identify possible areas of transformation. Admittedly, our data contrasts with results from a GALLIUM substudy which evaluated patients with PET imaging and showed overall transformation in CT staged patients was 2.5\% and high uptake on PET did not predict transformation. [37]

Limitations of this study include its retrospective nature, and the histological transformation rate may also be underestimated due to the lack of systemic biopsied at the time of progression. In addition, the blunted prognostic value of PFS24 in the PET era can be partly attributed from the evolved treatment paradigm in recent years. Better outcomes were observed from patients in the FOLL05 compared to the MSKCC cohorts. We hypothesized that the survival difference between the MSKCC vs FOLL05 cohorts may be caused by the nuances in the patient population. For example, less patients in the FOLL05 cohorts had high-risk FLIPI ( $37 \%$ vs $53 \%$ ) or elevated LDH (20\% vs 36\%). In addition, patients treated in the FOLL05 trial were enrolled onto a clinical trial where required centrally reviewed biopsies confirmed follicular lymphoma pathology, and a better survival could be expected by excluding de novo transformed biology. Both PET and CT imaged cohorts demonstrated similar absolute rate of PFS24 failures. Ultimately, our patients were treated with a wide range of first line and maintenance strategies over 10 years therefore evaluating the absolute rate of PFS24 failure may provide less nuance than understanding the timing and transformation status of the relapses. The existing data is also not suitable to associate PET 
utilization at time of treatment with identification of transformation prior to treatment. However, this may be an important question to address in the future.

Our study reveals staging with PET imaging at diagnosis and treatment may provide valuable information that impacts the outcomes of FL patients. Management of patients with early progression without histologic transformation needs to be better studied as this population demonstrates a more favorable prognosis as compared to patients with early progression and transformation of disease. Our study supports the incorporation of PET imaging to guide clinical decisions in the management of patients with FL. Future studies are needed to prospectively identify patients at risk for early progression and disease transformation, and design pre-emptive therapy aimed at improving treatment outcome and OS.

\section{Supplementary Material}

Refer to Web version on PubMed Central for supplementary material.

\section{Acknowledgements}

This work was supported in part by NCI Cancer Center Support Grant (P30 CA008748)

Declaration of Interests

1) Connie L. Batlevi: Research support: Janssen, Novartis, Epizyme, Xynomics, Bayer; Honoraria: Dava Oncology; Consulting: Kite, Juno. 2) Fushen Sha: None. 3) Anna Alperovich: None. 4) Andy Ni: None. 5) Katy Smith: None. 6) Zhitao Ying: None. 7) John F. Gerecitano: Currently employed by Janssen; Honoraria: Bayer, Epizyme, Roche, Genentech, Abbvie. 8) Paul A. Hamlin: Research support: Portola, Molecular Templates, Incyte, J\&J Pharmaceuticals; Consulting: Portola Pharmaceutics, Celgene, Karyopharm, Juno Therapeutics. 9) Steve M. Horwitz: Research support: ADCT therapeutics, Aileron,Celgene, Forty-Seven, Infinity/Verastem, Kyowa-HakkaKirin, Millenium/Takeda, Seattle Genetics, Trillium; Consulting ADCT therapeutics, Aileron,Corvus, Forty-Seven, Innate Pharma, Kyowa-Hakka-Kirin, Millenium/Takeda, Mundipharma, Portola, Seattle Genetics. 10) Anita Kumar: Research support: Abbvie, Adaptive Biotechnologies, Celgene, Pharmacyclics, Seattle Genetics; Scientific advisory board: Celgene. 11) Matthew J. Matasar: Research support: Genentech, Roche, GlaxoSmithKline, Bayer, Pharmacyclics, Janssen, Rocket Medical, Seattle Genetics; Honoraria: Genentech, Roche, Bayer, Pharmacyclics, Janssen, Seattle Genetics, GlaxoSmithKline. Consulting: Genentech, Bayer, Merck, Juno, Roche, Teva, Rocket Medical, Seattle Genetics; Consulting: Genentech, Roche, Seattle Genetics, Bayer. 12) Alison J. Moskowitz: Research support: Incyte, Seattle Genetics, BMS, and Merck: Consulting: Kyowa Hakko Kirin Pharma, Miragen Therapeutics, Takeda Pharmaceuticals, ADC therapeutics, Seattle Genetics, Cell Medica, Bristol-Myers Squibb, Erytech Pharma. 13) Craig H. Moskowitz: Research support: BMS, Merck, Seattle Genetics; Consulting: Astra Zeneca, BMS, Karyopharm Therapeutics, Merck, Seattle Genetics, Takeda, Vaniam Group; Scientific Advisory Board: Astra-Zeneca, Karyopharm Therapeutics, Merck, Seattle Genetics, Takeda, Vaniam Group. 14) Ariela Noy: Research support: Pharmacyclics, NIH, Raphael Pharma; Consulting: Janssen, Pharmacyclics, Medscape, Targeted Oncology. 15) Colette Owens: None. 16) Maria Lia M. Palomba: Research support: Genentech, Juno, Regeneron; Honoraria: Novartis, Merck, Celgene, Juno and Pharmacyclics; Consulting: None. 17) David Straus: Research support and Scientific advisory board: Seattle Genetics. 18) Gottfried von Keudell: Research support: Pharmacyclics, Genentech and Bayer. 19) Erel Joffe: None. 20) Andrew D. Zelenetz: Research support: MEI Pharma, MorphoSys, Sandoz, Celgene, Roche, Gilead; Consulting: Genentech/Roche, Gilead, Celgene, Janssen, Amgen, Novartis, Adaptive Biotechnology; Board of Directors (DMC Chair) Beigene. 21) Venkatraman E Seshan: None. 22) Stefano Luminari: Consulting and Scientific advisory board: Roche, Celgene, Sandoz, Gilead. 23) Luigi Marcheselli: None. 24) Massimo Federico: Research support: Mundipharma s.r.l., Chephalon/Teva, Celgene, Millennium/Takeda, and Roche; Consulting and scientific advisory boards: Takeda, Roche, Celgene, Sandoz, and Spectrum. 25) Anas Younes: Research support: Janssen, Curis, Merck, BMS, Syndax, and Roche; Honorarium: Janssen, AbbVie, Merck, Curis, Epizyme, Roche, Takeda; Consulting: Biopath, Xynomics, Epizyme, Roche. 


\section{References}

[1]. Morton LM, Wang SS, Devesa SS, Hartge P, Weisenburger DD, Linet MS. Lymphoma incidence patterns by WHO subtype in the United States, 1992-2001. Blood. 2006;107:265-76. [PubMed: 16150940]

[2]. Siegel RL, Miller KD, Jemal A. Cancer statistics, 2015. CA: A Cancer Journal for Clinicians. 2015;65:5-29. [PubMed: 25559415]

[3]. Casulo C, Byrtek M, Dawson KL, Zhou X, Farber CM, Flowers CR, et al. Early Relapse of Follicular Lymphoma After Rituximab Plus Cyclophosphamide, Doxorubicin, Vincristine, and Prednisone Defines Patients at High Risk for Death: An Analysis From the National LymphoCare Study. Journal of Clinical Oncology. 2015;33:2516-22. [PubMed: 26124482]

[4]. Maurer MJ, Bachy E, Ghesquiéres H, Ansell SM, Nowakowski GS, Thompson CA, et al. Early event status informs subsequent outcome in newly diagnosed follicular lymphoma. American journal of hematology. 2016;91:1096-101. [PubMed: 27465588]

[5]. Casulo C, Byrtek M, Dawson KL, Zhou X, Farber CM, Flowers CR, et al. Early Relapse of Follicular Lymphoma After Rituximab Plus Cyclophosphamide, Doxorubicin, Vincristine, and Prednisone Defines Patients at High Risk for Death: An Analysis From the National LymphoCare Study. J Clin Oncol. 2015;33:2516-22. [PubMed: 26124482]

[6]. Lansigan F, Barak I, Pitcher B, Jung SH, Cheson BD, Czuczman M, et al. The prognostic significance of PFS24 in follicular lymphoma following firstline immunotherapy: A combined analysis of 3 CALGB trials. Cancer Med. 2019;8:165-73. [PubMed: 30575311]

[7]. Casulo C, Barr PM. How I treat early-relapsing follicular lymphoma. Blood. 2019;133:1540-7. [PubMed: 30700421]

[8]. Wöhrer S, Jaeger U, Kletter K, Becherer A, Hauswirth A, Turetschek K, et al. 18F-fluoro-deoxyglucose positron emission tomography (18F-FDG-PET) visualizes follicular lymphoma irrespective of grading. Annals of Oncology. 2006;17:780-4. [PubMed: 16497824]

[9]. Luminari S, Biasoli I, Arcaini L, Versari A, Rusconi C, Merli F, et al. The use of FDG-PET in the initial staging of 142 patients with follicular lymphoma: a retrospective study from the FOLL05 randomized trial of the Fondazione Italiana Linfomi. Annals of Oncology. 2013;24:2108-12. [PubMed: 23585513]

[10]. Le Dortz L, De Guibert S, Bayat S, Devillers A, Houot R, Rolland Y, et al. Diagnostic and prognostic impact of 18F-FDG PET/CT in follicular lymphoma. European Journal of Nuclear Medicine and Molecular Imaging. 2010;37:2307-14. [PubMed: 20717826]

[11]. Metser U, Dudebout J, Baetz T, Hodgson DC, Langer DL, MacCrostie P, et al. [(18) F]-FDG PET/CT in the staging and management of indolent lymphoma: A prospective multicenter PET registry study. Cancer. 2017;123:2860-6. [PubMed: 28295218]

[12]. Trotman J, Barrington SF, Belada D, Meignan M, MacEwan R, Owen C, et al. Prognostic value of end-of-induction PET response after first-line immunochemotherapy for follicular lymphoma (GALLIUM): secondary analysis of a randomised, phase 3 trial. Lancet Oncol. 2018;19:1530 42. [PubMed: 30309758]

[13]. Karam M, Novak L, Cyriac J, Ali A, Nazeer T, Nugent F. Role of fluorine-18 fluorodeoxyglucose positron emission tomography scan in the evaluation and follow-up of patients with low-grade lymphomas. Cancer. 2006;107:175-83. [PubMed: 16721817]

[14]. Bodet-Milin C, Kraeber-Bodere F, Moreau P, Campion L, Dupas B, Le Gouill S. Investigation of FDG-PET/CT imaging to guide biopsies in the detection of histological transformation of indolent lymphoma. Haematologica. 2008;93:471-2. [PubMed: 18310543]

[15]. Noy A, Schoder H, Gonen M, Weissler M, Ertelt K, Cohler C, et al. The majority of transformed lymphomas have high standardized uptake values (SUVs) on positron emission tomography (PET) scanning similar to diffuse large B-cell lymphoma (DLBCL). Ann Oncol. 2009;20:50812. [PubMed: 19139176]

[16]. Schoder H, Noy A, Gonen M, Weng L, Green D, Erdi YE, et al. Intensity of 18fluorodeoxyglucose uptake in positron emission tomography distinguishes between indolent and aggressive non-Hodgkin's lymphoma. J Clin Oncol. 2005;23:4643-51. [PubMed: 15837966] 
[17]. Federico M, Luminari S, Dondi A, Tucci A, Vitolo U, Rigacci L, et al. R-CVP versus R-CHOP versus R-FM for the initial treatment of patients with advanced-stage follicular lymphoma: results of the FOLL05 trial conducted by the Fondazione Italiana Linfomi. J Clin Oncol. 2013;31:1506-13. [PubMed: 23530110]

[18]. Luminari S, Ferrari A, Manni M, Dondi A, Chiarenza A, Merli F, et al. Long-Term Results of the FOLL05 Trial Comparing R-CVP Versus R-CHOP Versus R-FM for the Initial Treatment of Patients With Advanced-Stage Symptomatic Follicular Lymphoma. J Clin Oncol. 2018;36:68996. [PubMed: 29095677]

[19]. Anderson JR, Cain KC, Gelber RD. Analysis of survival by tumor response. J Clin Oncol. 1983;1:710-9. [PubMed: 6668489]

[20]. Gray RJ. A Class of K-Sample Tests for Comparing the Cumulative Incidence of a Competing Risk. Ann Stat. 1988;16:1141-54.

[21]. Team RC. R: A language and environment for statistical computing. R Foundation for Statistical Computing Vienna, Austria2013.

[22]. Sarkozy C, Trneny M, Xerri L, Wickham N, Feugier P, Leppa S, et al. Risk Factors and Outcomes for Patients With Follicular Lymphoma Who Had Histologic Transformation After Response to First-Line Immunochemotherapy in the PRIMA Trial. J Clin Oncol. 2016;34:257582. [PubMed: 27298402]

[23]. Montoto S, Davies AJ, Matthews J, Calaminici M, Norton AJ, Amess J, et al. Risk and clinical implications of transformation of follicular lymphoma to diffuse large B-cell lymphoma. J Clin Oncol. 2007;25:2426-33. [PubMed: 17485708]

[24]. Bastion Y, Sebban C, Berger F, Felman P, Salles G, Dumontet C, et al. Incidence, predictive factors, and outcome of lymphoma transformation in follicular lymphoma patients. J Clin Oncol. 1997;15:1587-94. [PubMed: 9193357]

[25]. Federico M, Caballero Barrigon MD, Marcheselli L, Tarantino V, Manni M, Sarkozy C, et al. Rituximab and the risk of transformation of follicular lymphoma: a retrospective pooled analysis. Lancet Haematol. 2018;5:e359-e67. [PubMed: 30078408]

[26]. Cheah CY, Chihara D, Ahmed M, Davis RE, Nastoupil LJ, Phansalkar K, et al. Factors influencing outcome in advanced stage, low-grade follicular lymphoma treated at MD Anderson Cancer Center in the rituximab era. Ann Oncol. 2016;27:895-901. [PubMed: 26802151]

[27]. Morschhauser F, Salles G, Le Gouill S, Tilly H, Thieblemont C, Bouabdallah K, et al. An openlabel phase $1 \mathrm{~b}$ study of obinutuzumab plus lenalidomide in relapsed/refractory follicular B-cell lymphoma. Blood. 2018;132:1486-94. [PubMed: 30068505]

[28]. Dreyling M, Giurescu M, Grunert J, Fittipaldi F, Cupit L, Childs BH. CHRONOS-1: Open-label, uncontrolled phase II trial of intravenous phosphatidylinositol-3 kinase alpha/delta inhibitor copanlisib in patients with relapsed, indolent Non-Hodgkin's lymphomas (iNHL). Cancer Res. 2015;75.

[29]. Sehn LH, Chua N, Mayer J, Dueck G, Trneny M, Bouabdallah K, et al. Obinutuzumab plus bendamustine versus bendamustine monotherapy in patients with rituximab-refractory indolent non-Hodgkin lymphoma (GADOLIN): a randomised, controlled, open-label, multicentre, phase 3 trial. Lancet Oncol. 2016;17:1081-93. [PubMed: 27345636]

[30]. Gopal AK, Kahl BS, de Vos S, Wagner-Johnston ND, Schuster SJ, Jurczak WJ, et al. PI3Kdelta inhibition by idelalisib in patients with relapsed indolent lymphoma. N Engl J Med. 2014;370:1008-18. [PubMed: 24450858]

[31]. Andorsky DJ, Yacoub A, Bitran JD, Melear J, Brooks HD, Foon KA, et al. MAGNIFY: Phase IIIb Randomized Study of Lenalidomide Plus Rituximab (R) Followed By Lenalidomide Vs. Rituximab Maintenance in Subjects with Relapsed/Refractory Follicular, Marginal Zone, or Mantle Cell Lymphoma. Blood. 2016;128. [PubMed: 27827828]

[32]. Dreyling M, Morschhauser F, Bouabdallah K, Bron D, Cunningham D, Assouline SE, et al. Phase II study of copanlisib, a PI3K inhibitor, in relapsed or refractory, indolent or aggressive lymphoma. Ann Oncol. 2017;28:2169-78. [PubMed: 28633365]

[33]. Gopal AK, Kahl BS, Flowers CR, Martin P, Ansell SM, Abella-Dominicis E, et al. Idelalisib is effective in patients with high-risk follicular lymphoma and early relapse after initial chemoimmunotherapy. Blood. 2017;129:3037-9. [PubMed: 28325864] 
[34]. Leonard JP, Jung SH, Johnson J, Pitcher BN, Bartlett NL, Blum KA, et al. Randomized Trial of Lenalidomide Alone Versus Lenalidomide Plus Rituximab in Patients With Recurrent Follicular Lymphoma: CALGB 50401 (Alliance). J Clin Oncol. 2015;33:3635-40. [PubMed: 26304886]

[35]. Martin Dreyling AS, Mollica Luigina, Leppä Sirpa, Follows George A, Lenz Georg, Kim Won Seog, , Nagler Arnon, Panayiotidis Panayiotis, Demeter Judit, Dr.Sci, Özcan Muhit, Kosinova Marina,, Bouabdallah Krimo, Morschhauser Franck, Stevens Don, Trevarthen David, Rodrigues Liana, Hiemeyer Florian, Wang MingLu, Garcia-Vargas Jose, Childs Barrett H., and Zinzani Pier Luigi. Long-Term Efficacy and Safety from the Copanlisib CHRONOS-1 Study in Patients with Relapsed or Refractory Indolent B-Cell Lymphoma. ASH San Diego2018

[36]. Freeman CL, Kridel R, Moccia AA, Savage KJ, Villa DR, Scott DW, et al. Early progression after BR is associated with high risk of transformation in advanced stage follicular lymphoma. Blood. 2019.

[37]. Mir F, Barrington SF, Meignan M, Brown H, Nielsen T, Sahin D, et al. Baseline Suvmax Did Not Predict Histological Transformation from Follicular Lymphoma to Aggressive Lymphoma in the Phase III GALLIUM Study. Blood. 2018;132:4160-. 


\section{Research in context}

\section{Evidence before this study}

We searched PubMed on May 28, 2019 with no date restrictions for all original research (i.e., excluding reviews) with the terms "follicular lymphoma" in the title, and "outcomes" in the title or abstract. This search identified 173 articles. Three studies have suggested that early progression within 2 years of initiating first-line treatment or initial diagnosis in patients with follicular lymphoma predicted a poor overall survival. However, the definition of early disease progression, varied among studies. Furthermore, the imaging modalities used in these reports were not specified. We found no reports of the prognostic value of early progression of disease in the modern PET staging era.

\section{Added value of this study}

We report on the role of imaging modality used for staging patients with FL on the prognostic impact of early progression of disease (POD). Consistent with previous reports, we found that early POD in CT-staged patients to confer a poor overall survival (OS). However, when modern PET-based staging was used, the impact of early POD on OS was diminished. Our results suggest that the poor prognosis associated with early POD is predominantly driven by early histologic transformation which can be missed before initiating therapy in CT-staged patients. When modern PET-imaging was used before initiating therapy, early POD was less frequently associated with histologic transformation, and therefore early POD had a lower impact on OS.

\section{Implications of all the available evidence}

Our study demonstrates that in the modern PET-staging era, early POD with histologic transformation continues to confer a poor prognosis requiring novel treatment approaches. In contrast, patients with early POD but with no histologic transformation have a good outcome with standard therapy and can be spared from intensive and more toxic therapy. Our study highlights the need for repeated biopsies in patients with early POD to guide treatment decisions. 


\section{Highlights:}

- $\quad$ Early progression after first line treatment in follicular lymphoma is a marker of poor prognosis

- $\quad$ PET staging possibly identifies patients with highest risk of poor outcomes

- $\quad$ PET staging reduces the prognostic impact of early progression likely by excluding patients with early transformation

- $\quad$ Patients with early progression and early transformation have the highest risk of poor outcomes 


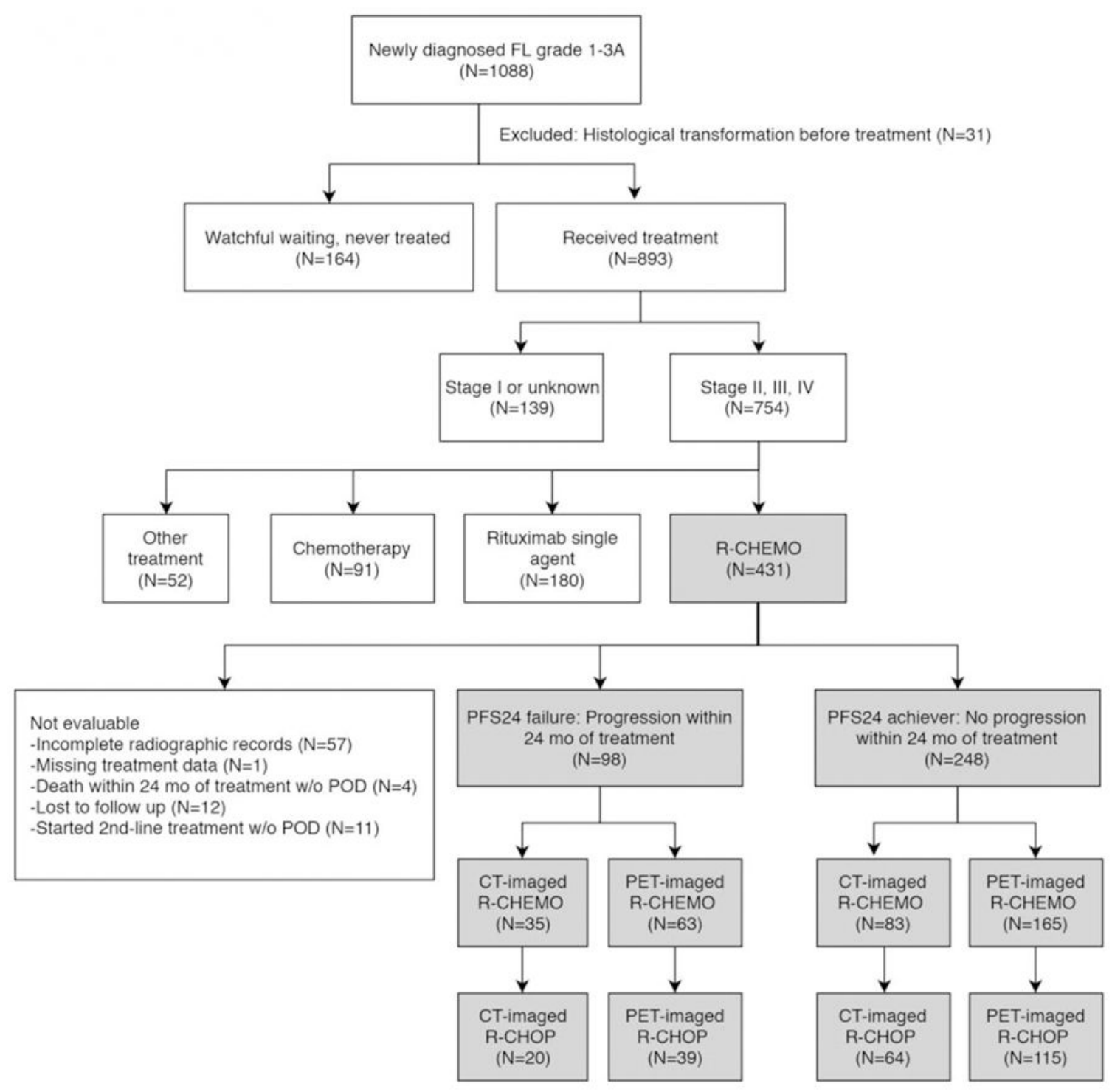

Figure 1.

Consort diagram of patients at initial treatment who were evaluable for progression-free survival (PFS) status at 24 months in the MSKCC cohort. 


\section{A. MSKCC R-CHOP Event defining analysis}

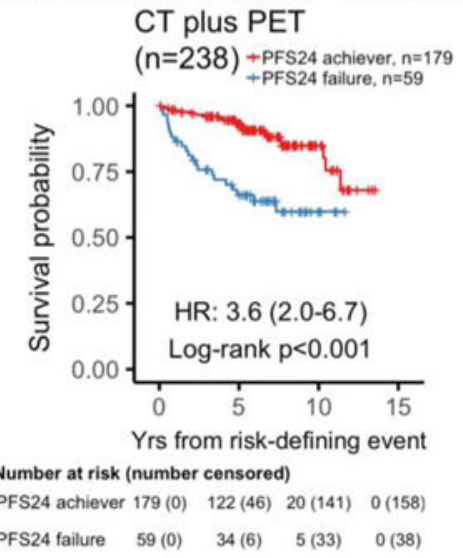

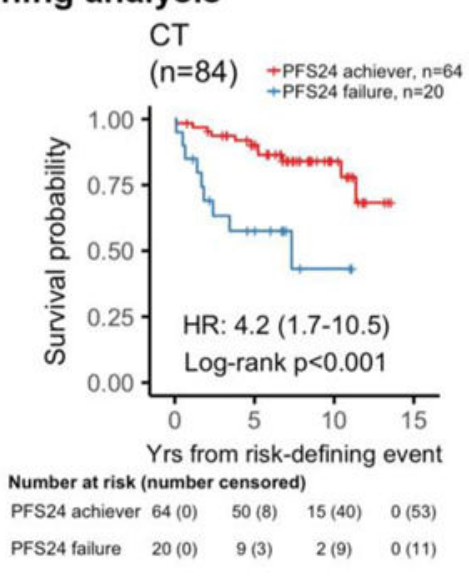

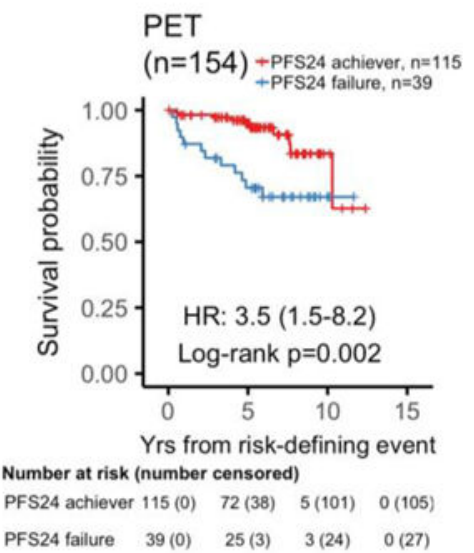

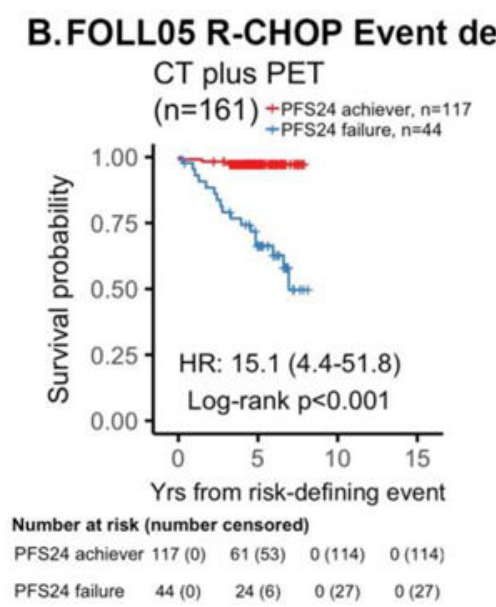
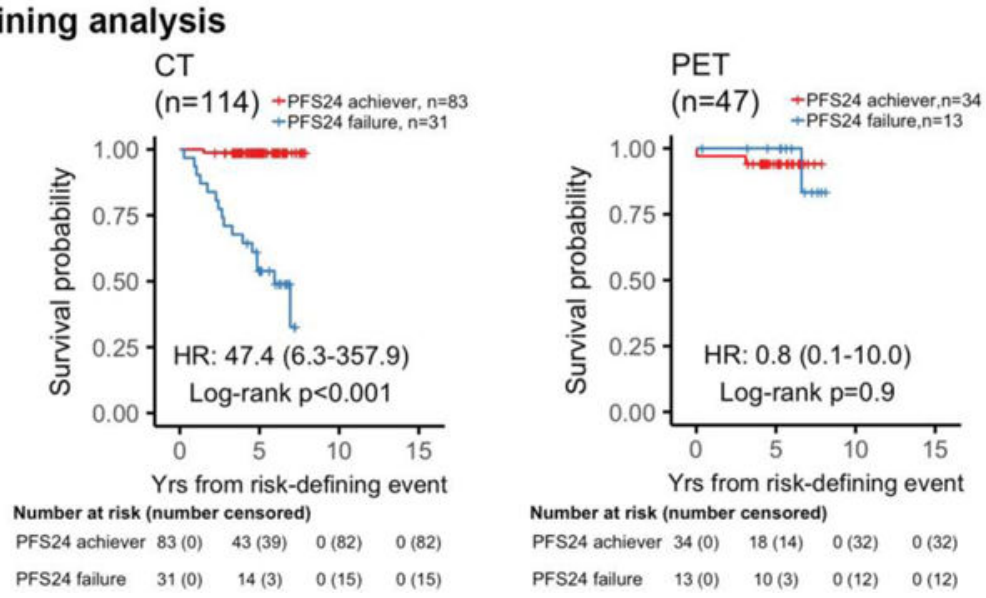

Figure 2. Event defining analysis of PFS24 based on radiographic imaging at time of treatment in patients treated with R-CHOP.

Kaplan-Meier plots of overall survival (OS) since risk-defining event stratified by PFS24 status in patients with stage II-IV disease treated with R-CHOP. (A) MSKCC cohort. (B) Validation FOLL05 cohort. When comparing OS by PFS24 status, PFS24 failures staged by CT had a worse outcome than those who were staged with PET. For CT-staged patients who failed PFS24, the estimated 10-year OS rate was $43 \%$ versus PET-staged that was $67 \%$. The results were reproduced in the validation cohort where CT-imaged PFS24 failures had an estimated 5-year OS rate of 54\% versus PET-imaged PFS24 failures had an estimated 5-year OS rate of $100 \%$. 


\section{A. MSKCC R-CHOP Landmark analysis}

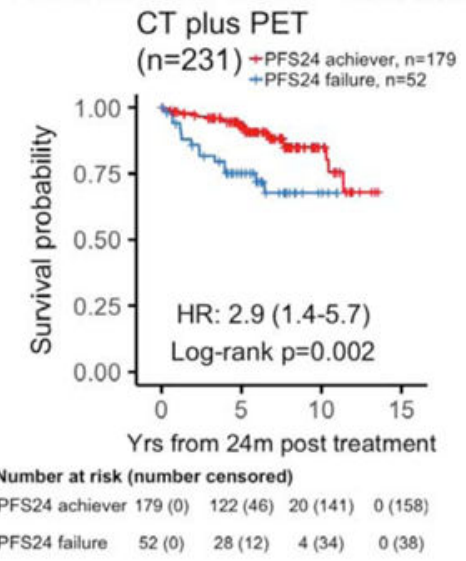

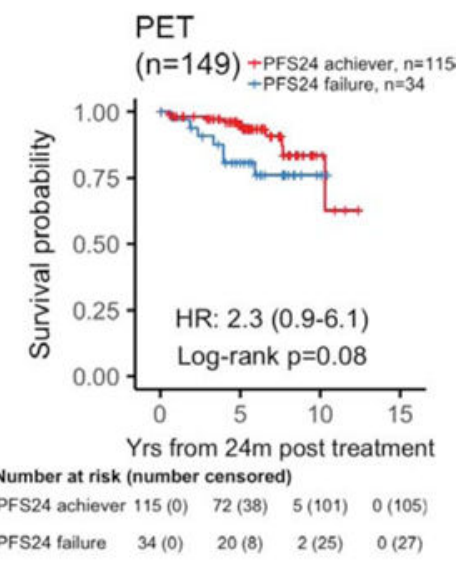

\section{B.FOLL05 R-CHOP Landmark analysis}

CT plus PET

$(n=158)+$ PFS24 achiever, $n=117$

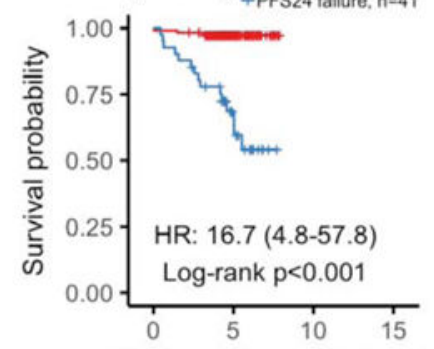

Yrs from $24 \mathrm{~m}$ post treatment Number at risk (number censored)

PFS24 achiever $117(0) \quad 61(53) \quad 0(114) \quad 0(114)$

PFS24 fallure $\quad 41(0) \quad 15(14) \quad 0(26) \quad 0(26)$

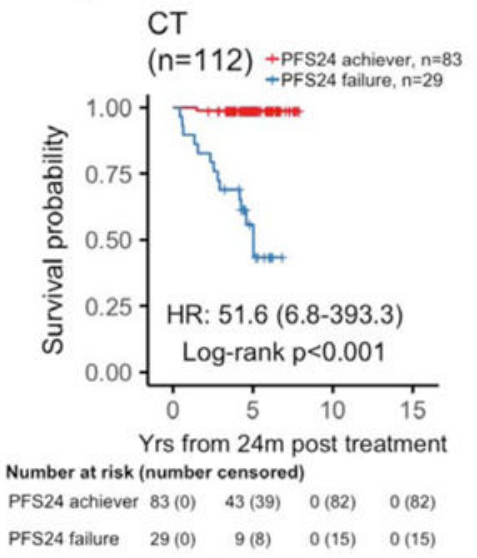

Figure 3. Landmark analysis of PFS24 based on radiographic imaging at time of treatment in patients treated with R-CHOP.

Kaplan-Meier plots of overall survival (OS) since 2 years landmark stratified by PFS24 status in patients with stage II-IV disease treated with R-CHOP. (A) MSKCC cohort. (B) Validation FOLL05 cohort. When comparing OS by PFS24 status, PFS24 failures staged by $\mathrm{CT}$ had a worse outcome than those who were staged with PET. For CT-staged patients who failed PFS24, the estimated 10-year OS rate was 47\% versus PET-staged that was $76 \%$. The results were reproduced in the validation cohort where CT-imaged PFS24 failures had an estimated 5-year OS rate of 56\% versus PET-imaged PFS24 failures had an estimated 5-year OS rate of $100 \%$. 


\section{A. MSKCC R-CHEMO Landmark analysis}

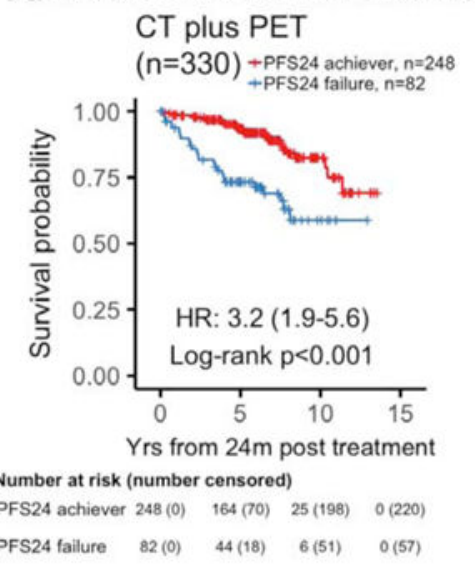

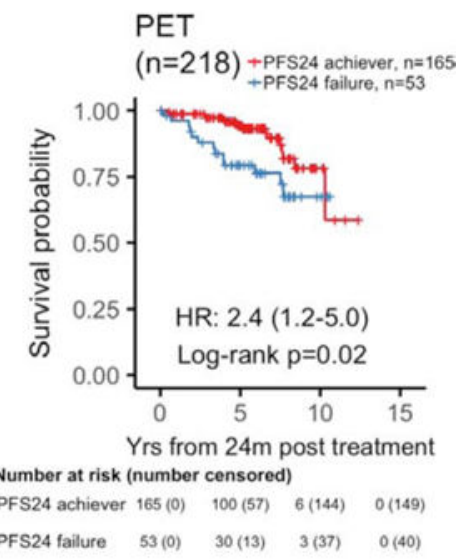

\section{B.FOLL05 R-CHEMO Landmark analysis}

CT plus PET

$(n=473)+$ PFS24 achiever, $n=339$

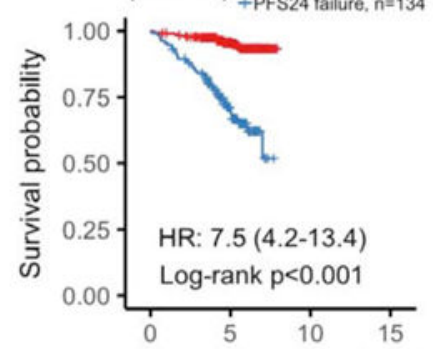

Yrs from $24 \mathrm{~m}$ post treatment

Number at risk (number censored)

PFS24 achiever $339(0) \quad 166(160) \quad 0(323) \quad 0(323)$

PFS24 failure $134(0) \quad 50(49) \quad 0(93) \quad 0(93)$

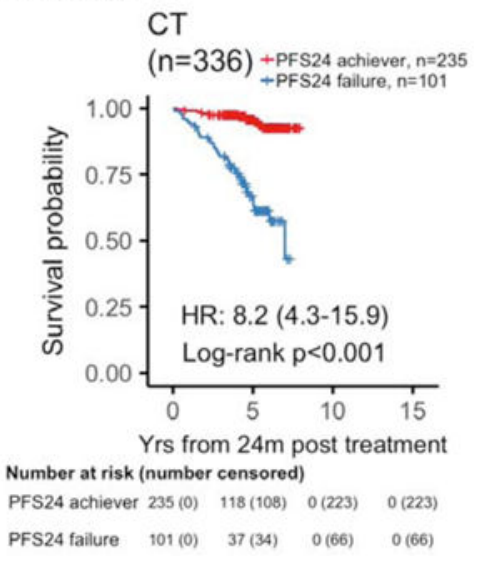

Figure 4. Landmark analysis of PFS24 based on radiographic imaging at time of treatment in patients treated with R-chemotherapy.

Kaplan-Meier plots of overall survival (OS) since 2 years landmark stratified by PFS24 status in patients with stage II-IV disease treated with rituximab plus chemotherapy (RChemo). (A) MSKCC cohort. (B) FOLL05 cohort. When comparing OS by PFS24 status, PFS24 failures staged by CT had a worse outcome than those who were staged with PET. For CT-staged patients who failed PFS24, the estimated 10-year OS rate was $44 \%$ versus PET-staged that was $67 \%$. The results were reproduced in the validation cohort where CTimaged PFS24 failures had a an estimated 5-year OS rate of 67\% versus PET-imaged PFS24 failures had an estimated 5-year OS rate of $84 \%$. 


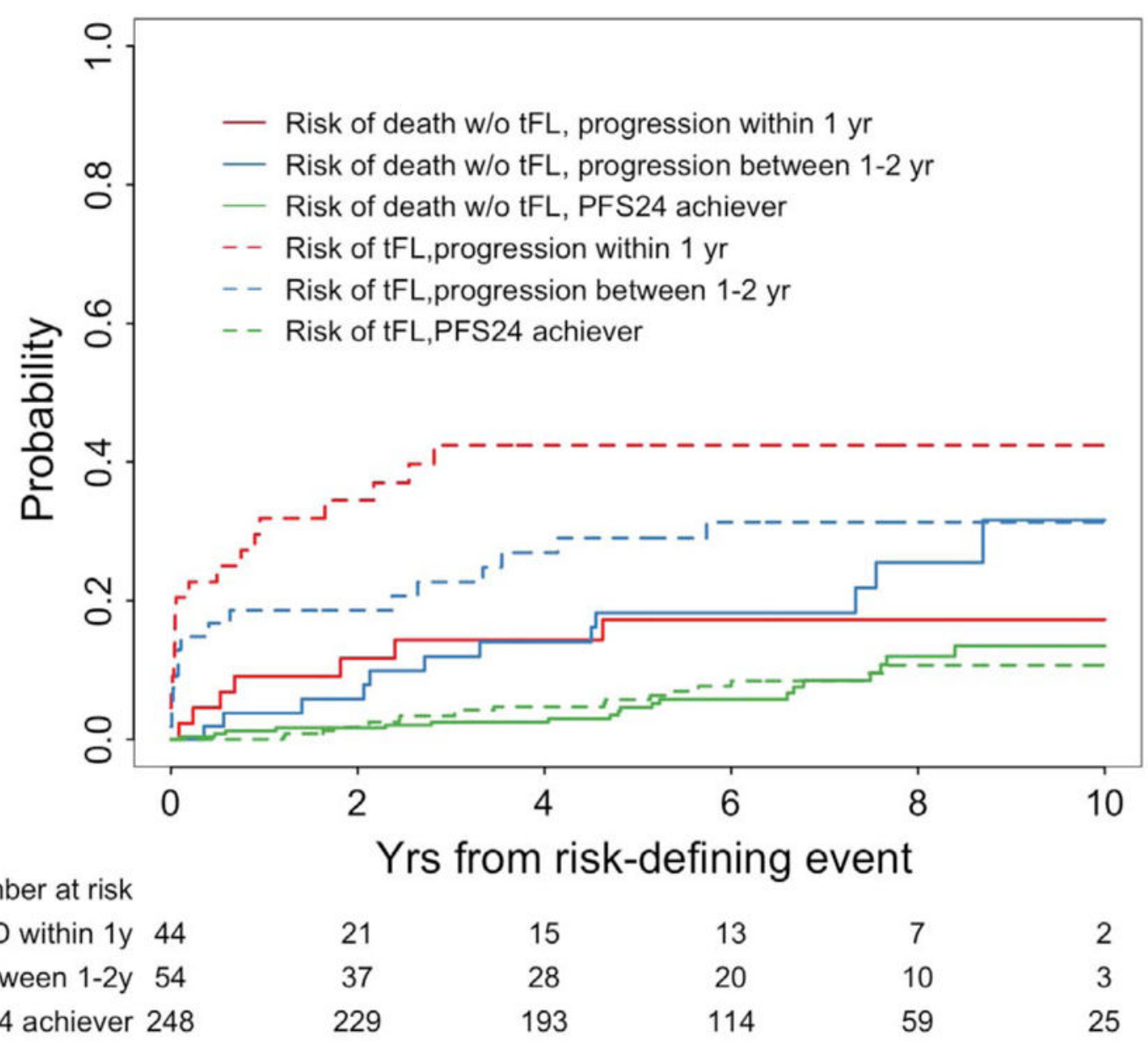

Figure 5. Competing risk analysis to assess association between early progression and transformation in MSKCC patients treated with R-chemotherapy.

Patients were categorized as early progressors within 1 year, early progressors between 1-2 years, and PFS24 achievers who lack progression within 2 years. The competing events were transformation or death without transformation. The risk of transformation based on time from risk defining event (dashed lines) were compared to the risk of death not attributed to transformation (solid lines). 
Table 1.

Patient characteristics at treatment initiation. In the MSKCC cohort, 346 patients with stage II-IV disease treated with R-Chemo were evaluable for PFS24 status after first line treatment. In the validation FOLL05 Cohort, 484 patients with stage II-IV disease treated with R-Chemo were evaluable for PFS24 status after first line treatment.

\begin{tabular}{|c|c|c|c|c|c|c|c|c|c|c|}
\hline \multirow[b]{3}{*}{ Characteristic } & \multicolumn{5}{|c|}{$\operatorname{MSKCC}(\mathbf{N}=346)$} & \multicolumn{5}{|c|}{ FOLL05 $(\mathrm{N}=484)$} \\
\hline & \multicolumn{2}{|c|}{ CT staged (N=118) } & \multicolumn{3}{|c|}{ PET staged $(n=228)$} & \multicolumn{2}{|c|}{ CT staged $(\mathrm{N}=345)$} & \multicolumn{3}{|c|}{ PET staged $(n=139)$} \\
\hline & No. & $\%$ & No. & $\%$ & $P$ & No. & $\%$ & No. & $\%$ & $P$ \\
\hline Age: median (Range) & \multicolumn{2}{|c|}{$56(24-80)$} & \multicolumn{2}{|c|}{$55(23-85)$} & $0 \cdot 95$ & \multicolumn{2}{|c|}{$55(29-75)$} & \multicolumn{2}{|c|}{$56(32-74)$} & $0 \cdot 24$ \\
\hline \multicolumn{11}{|l|}{ Gender } \\
\hline Female & 54 & $46 \%$ & 99 & $43 \%$ & \multirow{2}{*}{0.73} & 164 & $48 \%$ & 72 & $52 \%$ & \multirow{2}{*}{0.42} \\
\hline Male & 64 & $54 \%$ & 129 & $57 \%$ & & 181 & $52 \%$ & 67 & $48 \%$ & \\
\hline \multicolumn{11}{|l|}{ Stage } \\
\hline II & 6 & $5 \%$ & 13 & $6 \%$ & \multirow{2}{*}{1.00} & 33 & $10 \%$ & 9 & $6 \%$ & \multirow{2}{*}{$0 \cdot 37$} \\
\hline $\mathrm{III} / \mathrm{IV}$ & 112 & $95 \%$ & 215 & $94 \%$ & & 312 & $90 \%$ & 130 & $94 \%$ & \\
\hline \multicolumn{11}{|l|}{ FLPI } \\
\hline Low & 15 & $16 \%$ & 24 & $12 \%$ & & 74 & $21 \%$ & 31 & $22 \%$ & \\
\hline Intermediate & 30 & $32 \%$ & 66 & $34 \%$ & 0.69 & 144 & $42 \%$ & 57 & $41 \%$ & 0.98 \\
\hline High & 49 & $52 \%$ & 105 & $54 \%$ & & 127 & $37 \%$ & 51 & $37 \%$ & \\
\hline Unknown & \multicolumn{2}{|c|}{24} & \multicolumn{2}{|c|}{33} & & & & & & \\
\hline \multicolumn{11}{|l|}{$\mathrm{LDH}$} \\
\hline Elevated & 31 & $35 \%$ & 68 & $37 \%$ & & 67 & $19 \%$ & 28 & $20 \%$ & \multirow{2}{*}{0.90} \\
\hline Normal & 57 & $65 \%$ & 117 & $63 \%$ & $0 \cdot 89$ & 278 & $81 \%$ & 111 & $80 \%$ & \\
\hline Unknown & \multicolumn{2}{|c|}{30} & \multicolumn{2}{|c|}{43} & & & & & & \\
\hline \multicolumn{11}{|l|}{ Treatment } \\
\hline R-CHOP & 84 & $71 \%$ & 154 & $68 \%$ & \multirow{5}{*}{0.93} & 114 & $33 \%$ & 47 & $34 \%$ & \\
\hline R-CVP & 21 & $18 \%$ & 41 & $18 \%$ & & 116 & $34 \%$ & 43 & $31 \%$ & 0.85 \\
\hline R-FM & 1 & $1 \%$ & 4 & $2 \%$ & & 115 & $33 \%$ & 49 & $35 \%$ & \\
\hline R-Bendamustine & 6 & $5 \%$ & 14 & $6 \%$ & & & & & & \\
\hline Other & 6 & $5 \%$ & 15 & $7 \%$ & & & & & & \\
\hline Rituximab maintenanc & & & & & & & & & & \\
\hline Yes & 27 & $23 \%$ & 74 & $32 \%$ & & 0 & $0 \%$ & 0 & $0 \%$ & 1.00 \\
\hline No & 91 & $77 \%$ & 154 & $68 \%$ & & 345 & $100 \%$ & 139 & $100 \%$ & 1.00 \\
\hline Subsequent HDT/SCT & & & & & & & & & & \\
\hline Autologous & 7 & $6 \%$ & 20 & $9 \%$ & & - & & - & & \\
\hline Allogeneic & 6 & $5 \%$ & 12 & $5 \%$ & 0.84 & & & & & \\
\hline Both & 3 & $3 \%$ & 7 & $3 \%$ & & & & & & \\
\hline No & 102 & $86 \%$ & 189 & $83 \%$ & & - & & - & & \\
\hline PFS24 status & & & & & & & & & & \\
\hline PFS24 achievers & 83 & $70 \%$ & 165 & $72 \%$ & 071 & 235 & $68 \%$ & 104 & $75 \%$ & 016 \\
\hline PFS24 failures & 35 & $30 \%$ & 63 & $28 \%$ & 0.11 & 110 & $32 \%$ & 35 & $25 \%$ & 0.16 \\
\hline
\end{tabular}

Eur J Cancer. Author manuscript; available in PMC 2021 February 01. 
Abbreviations: FLIPI, Follicular Lymphoma International Prognostic Index; HDT/SCT, high-dose therapy and stem cell transplant; LDH, lactate dehydrogenase; R-CHOP, rituximab plus cyclophosphamide, doxorubicin, vincristine, and prednisone; R-CVP, rituximab plus cyclophosphamide, vincristine, and prednisone; R-FM, rituximab plus fludarabine and mitoxantrone 
Table 2.

Rate of histological transformation and risk of death based on PFS24 status. Risk of transformed FL denotes the culminative risk of biopsy-proven transformation at the time of the relapse. The culminative risk of death without transformation is provided using a competing risk analysis. The risk of death due to transformation is calculated by the total risk of death minus the risk of death without transformation.

\begin{tabular}{|c|c|c|c|c|}
\hline & $\begin{array}{c}\text { Years after } \\
\text { risk-defining } \\
\text { event }\end{array}$ & $\begin{array}{c}\text { Progression within } 1 \\
\text { year }(n=44) ; \\
\text { cumulative risk } \\
\text { (cumulative cases) }\end{array}$ & $\begin{array}{l}\text { Progression between } \\
\text { 1-2 year }(\mathrm{n}=54) ; \\
\text { cumulative risk } \\
\text { (cumulative cases) }\end{array}$ & $\begin{array}{l}\text { PFS24 achievers } \\
\text { (n=248); cumulative } \\
\text { risk (cumulative } \\
\text { cases) }\end{array}$ \\
\hline \multirow{4}{*}{ Risk of $\mathrm{tFL}, \%$} & 0.02 (1 week) & $9 \cdot 1(4)$ & $5 \cdot 6(3)$ & $0 \cdot 0(0)$ \\
\hline & 1 & $31.9(14)$ & $18 \cdot 6(10)$ & $0 \cdot 0(0)$ \\
\hline & 2 & $34 \cdot 5(15)$ & $18 \cdot 6(10)$ & $2 \cdot 1(5)$ \\
\hline & 3 & $42 \cdot 4(18)$ & $22 \cdot 7(12)$ & $3 \cdot 4(8)$ \\
\hline \multirow{4}{*}{ Risk of death due to tFL, $\%$} & 0.02 (1 week) & $0 \cdot 0(0)$ & $0 \cdot 0(0)$ & $0 \cdot 0(0)$ \\
\hline & 1 & $11.4(5)$ & $5 \cdot 7(3)$ & $0 \cdot 0(0)$ \\
\hline & 2 & $16 \cdot 2(7)$ & $7 \cdot 7(4)$ & $0 \cdot 0(0)$ \\
\hline & 3 & $18 \cdot 6(8)$ & $9 \cdot 8(5)$ & $0 \cdot 9(2)$ \\
\hline \multirow{4}{*}{ Risk of death without $\mathrm{tFL}, \%$} & 0.02 (1 week) & $0 \cdot 0(0)$ & $0 \cdot 0(0)$ & $0 \cdot 0(0)$ \\
\hline & 1 & $9 \cdot 1(4)$ & $3 \cdot 8(2)$ & $1 \cdot 2(3)$ \\
\hline & 2 & $11 \cdot 7(5)$ & $5 \cdot 8(3)$ & $1.6(4)$ \\
\hline & 3 & $14 \cdot 4(6)$ & $12 \cdot 0(6)$ & $2 \cdot 5(6)$ \\
\hline \multirow{4}{*}{$\begin{array}{l}\text { Total risk of death (sum of risk of death } \\
\text { from tFL and without } \mathrm{tFL} \text { ), } \%\end{array}$} & 0.02 (1 week) & $0 \cdot 0(0)$ & $0 \cdot 0(0)$ & $0 \cdot 0(0)$ \\
\hline & 1 & $20.5(9)$ & $9 \cdot 4(5)$ & $1 \cdot 2(3)$ \\
\hline & 2 & $27 \cdot 8(12)$ & $13 \cdot 6(7)$ & $1 \cdot 6(4)$ \\
\hline & 3 & $33 \cdot 0(14)$ & $21 \cdot 8(11)$ & $3 \cdot 4(8)$ \\
\hline
\end{tabular}

